\title{
Post-Markov master equation for the dynamics of open quantum systems
}

\author{
Ting $\mathrm{Yu}^{1}$, Lajos Diósi ${ }^{2,3}$, Nicolas Gisin ${ }^{1}$, and Walter T. Strunz ${ }^{4}$ \\ ${ }^{1}$ Group of Applied Physics, University of Geneva, 1211 Geneva 4, Switzerland \\ ${ }^{2}$ Institute for Advanced Study, Wallotstrasse 19, D-14193 Berlin, Germany \\ ${ }^{3}$ Research Institute for Particle and Nuclear Physics, 1525 Budapest 114, POB 49, Hungary \\ ${ }^{4}$ Fachbereich Physik, Universität GH Essen, 45117 Essen, Germany
}

(May 3, 1999)

\begin{abstract}
A systematic first-order correction to the standard Markov master equation for open quantum systems interacting with a bosonic bath is presented. It extends the Markov Lindblad master equation to the more general case of non-Markovian evolution. The meaning and applications of our 'post'-Markov master equation are illustrated with several examples, including a damped twolevel atom, the spin-boson model and the quantum Brownian motion model. Limitations of the Markov approximation, the problem of positivity violation and initial slips are also discussed.
\end{abstract}

03.65.Bz Foundations, theory of measurement, miscellaneous theories 05.40.-a Fluctuation phenomena, random processes, noise, and Brownian motion 42.50.Lc Quantum fluctuations, quantum noise, and quantum jumps

The fundamental approach to open quantum systems relies on a closed system-plus-reservoir model whose time evolution is governed by the standard Schrödinger or von-Neumann equation. Due to the coupled dynamics of system and environment, effectively, the evolution of the system's reduced density operator $\rho_{t}$ depends on its past, it is nonMarkovian. For a quantum particle coupled to an environment of harmonic oscillators, this is obvious from its path integral propagator as derived by Feynman and Vernon [1]. It is also apparent from Zwanzig's projection approach [2] where the evolution equation for $\rho_{t}$ takes the form of an integro-differential equation involving an integral over the whole history of the density operator. Exactly how far back the integration over the past has to be extended defines the environmental 'memory' time scale $\tau$.

A finite memory time $\tau$ leads to severe problems for the treatment of open system dynamics which is even more true for attempts to find numerical solutions. Growing experimental advances on mesoscopic scales [3], however, demand an efficient theory for open system dynamics beyond the standard Markov approximation: these are experiments with high-Q microwave cavities, investigations of the spontaneous emission from atoms in a structured radiation continuum (e.g. 'photonic band gap' materials) or the output coupling from a Bose-Einstein condensate to create an atom laser, to name a few. Also, the important phenomenon of decoherence which takes place on time scales that can be of the same order as the correlation time of the environment requires theories beyond the standard Markov approximation.

There are a few derivations of exact non-Markovian master equations for model systems (see e.g. [四 7]). Remarkably, despite the underlying non-Markovian dynamics, these exact evolution equations may be cast into the form of a timelocal master equation involving the reduced density operator $\rho_{t}$ at time $t$ only. A finite memory time and thus non-Markovian effects are exactly taken into account by suitable time dependent coefficients entering the resulting master equation.

The derivation of a useful master equation for general open systems beyond the Markov regime remains an outstanding open problem. In this Letter we present a first order 'post'-Markov master equation for an arbitrary quantum system coupled to an environment of harmonic oscillators. As in the case of the above-mentioned soluble models, our result takes the form of a time-local master equation with time dependent coefficients involving the bath correlation function. To zeroth order in the memory time $(\tau=0)$ we recover the standard Markov master equation.

To begin with, let us recall the Markov case. From an axiomatic approach, Lindblad [8] derived the most general Markov evolution equation for a density operator $(\hbar=1)$ :

$$
\dot{\rho}_{t}=-i\left[H, \rho_{t}\right]+\frac{1}{2}\left(\left[L, \rho_{t} L^{\dagger}\right]+\left[L \rho_{t}, L^{\dagger}\right]\right),
$$

where, for simplicity, we only consider the case of a single Lindblad operator $L$ representing the influence of the environment. Such Markov Lindblad master equations are widely used in quantum optics [9]. We identify two 'system' time scales in (1): the ordinary dynamic time scale determined by the Hamiltonian $H$ (which we call $\omega^{-1}$ ), and a damping or relaxation time scale (which we call $\gamma^{-1}$ ) determined by the operator $L^{\dagger} L$. The true open system dynamics will be well described by equation (11) as long as terms of the order $\omega \tau$ and $\gamma \tau$ can be neglected, where $\tau$ is again the environmental memory time. 
Our aim is a 'post'-Markov master equation valid to first order in $\omega \tau, \gamma \tau$. In what follows, we explain the main result and illustrate it with several prominent physical problems, including a damped two-level system, the spinboson model and the quantum Brownian motion model. We start with a quantum system interacting with a bosonic oscillator environment with total Hamiltonian,

$$
H_{\mathrm{tot}}=H+\sum_{\lambda} g_{\lambda}\left(L a_{\lambda}^{\dagger}+L^{\dagger} a_{\lambda}\right)+\sum_{\lambda} \omega_{\lambda} a_{\lambda}^{\dagger} a_{\lambda}
$$

Here, $H$ is the Hamiltonian of the system and $L$ a system operator describing the coupling to the environment. The standard zero temperature bath correlation function of model (2) is

$$
\alpha(t, s)=\sum_{\lambda} g_{\lambda}^{2} e^{-i \omega_{\lambda}(t-s)} .
$$

Its decay, as a function of the time delay $t-s$, defines the 'memory' or correlation time $\tau$ of the environment. We choose to normalize the coupling constants $g_{\lambda}$ in (2) such that $\alpha(t, s)$ is normalized $\left(\int \alpha(t, s) d s=1\right)$ which means that the overall coupling strength is determined by a parameter hidden in the coupling operator $L$ in (2).

A lengthy derivation [10] based on a non-Markovian quantum trajectory approach developed recently [11], shows that to first order in the memory time $\tau$ the reduced density operator of model (2) evolves according to the first-order 'post'-Markov master equation,

$$
\begin{aligned}
\dot{\rho}_{t}= & -i\left[H, \rho_{t}\right] \\
& +g_{0}(t)\left[L, \rho_{t} L^{\dagger}\right]+g_{0}^{*}(t)\left[L \rho_{t}, L^{\dagger}\right] \\
& +i g_{1}(t)\left[L^{\dagger},[H, L] \rho_{t}\right]-i g_{1}^{*}(t)\left[\rho_{t}\left[L^{\dagger}, H\right], L\right] \\
& +g_{2}(t)\left[L^{\dagger},\left[L^{\dagger}, L\right] L \rho_{t}\right]+g_{2}^{*}(t)\left[\rho_{t} L^{\dagger}\left[L^{\dagger}, L\right], L\right],
\end{aligned}
$$

where

$$
\begin{aligned}
& g_{0}(t)=\int_{0}^{t} \alpha(t, s) d s, \\
& g_{1}(t)=\int_{0}^{t} \alpha(t, s)(t-s) d s, \\
& g_{2}(t)=\int_{0}^{t} \int_{0}^{s} \alpha(t, s) \alpha(s, u)(t-s) d u d s .
\end{aligned}
$$

The master equation (何) is the central theme of this paper. Note first that according to our convention, the function $g_{0}(t)$ is of the order one. Therefore, as in (17), the first two lines in (四) define the time scales $\omega^{-1}(H)$ and $\gamma^{-1}\left(L^{\dagger} L\right)$. The two functions $g_{1}(t)$ and $g_{2}(t)$, however, are of the order of the memory time $\tau$. Therefore, the third and fourth line in (4) are smaller than the first and second line, respectively, by a factor $\gamma \tau$. As $\tau \rightarrow 0$, the third and forth line in (4) become negligible, the real part of $g_{0}(t)$ tends to $\frac{1}{2}$, and the contribution of the imaginary part of $g_{0}(t)$ can be absorbed by a renormalized Hamiltonian: our 'post'-Markov master equation (4) reduces to the Lindblad master equation (11) for vanishing memory time $\tau$. The third and fourth lines, which arise due to short but finite correlation time, represent the new first-order non-Markovian corrections. The time-dependent coefficients $g_{i}(t)$ describe an important initial slip on the time scale of the memory time $\tau$ 肺. Note that (何, assuming that the initial state is factorable, is valid for zero temperature and also for finite temperature if $L=L^{\dagger}$. General finite temperatue post-Markov master equation can be obtained direclty from the first order perturbation of the finite temperature quantum state diffusion(QSD) equation 11.

As a first example, we consider a damped two-level system: $H=\frac{\omega}{2} \sigma_{z}, L=\sqrt{\gamma} \sigma_{-}$. For the sake of simplicity, we phenomenologically choose an exponentially decaying correlation function $\alpha(t, s)=\frac{1}{2 \tau} \mathrm{e}^{-|t-s| / \tau}$ with memory time $\tau$. Since this model can be solved exactly [7.11], we are able to compare results from our post-Markov master equation (田)

$$
\begin{aligned}
\dot{\rho}_{t}= & -i \frac{\omega}{2}\left[\sigma_{z}, \rho_{t}\right]-i \omega\left(\gamma g_{1}(t)\right)\left[\sigma_{+} \sigma_{-}, \rho_{t}\right] \\
& +\gamma\left(g_{0}(t)+\gamma g_{2}(t)\right)\left(2 \sigma_{-} \rho_{t} \sigma_{+}\right. \\
& \left.-\left\{\sigma_{+} \sigma_{-}, \rho_{t}\right\}\right),
\end{aligned}
$$


with the exact result. It here happens that the post-Markov master equation (8) is of Lindblad form (1) with timedependent coefficients (the real $g_{1}(t)$ gives rise to a time-dependent frequency shift). Therefore we know that $\rho_{t}$ remains a proper density operator for all times and all parameters. As seen in the next two examples this property is not a generic feature of the post-Markov master equation (仼. To illustrate the limits of the Markov approximation we compare in Fig. 1 the average $\left\langle\sigma_{y}\right\rangle$ obtained from the post-Markov master equation (dashed curve), from the Markov master equation (dotted curve) and from the exact master equation (solid curve) for the parameters $\omega=\gamma$ and $\gamma \tau=0.2$. We see that the post-Markov master equation gives a much better result than the Markov master equation as expected for a significant memory time of $\gamma \tau=0.2$.

So far, our results assume a zero temperature environment. As stated before, it turns out [11], that for a selfadjoint coupling operator $L=L^{\dagger}$ the same analysis holds, with $\alpha(t, s)$ in (3) replaced by the finite temperature expression $\alpha(t, s)=\sum_{\lambda} g_{\lambda}^{2}\left[\operatorname{coth}\left(\frac{\omega_{\lambda}}{2 k T}\right) \cos \omega_{\lambda}(t-s)-i \sin \omega_{\lambda}(t-s)\right](\hbar=1)$. A finite temperature introduces an additional time scale $1 / k T$ but we here focus on the high-temperature limit $k T \gg \tau^{-1}$, thus disregarding the new time scale. For an Ohmic bath we obtain the standard correlation function

$$
\alpha(t, s)=2 k T \Delta(t-s)+i \dot{\Delta}(t-s)
$$

where $\Delta(t)$ is a smeared out delta function, decaying on the memory time scale $\tau$ (the inverse of the cutoff frequency), and $\dot{\Delta}$ is its time derivative. The precise shape of $\Delta(t)$ depends on the type of high-frequency cutoff chosen in the sum (3). Due to the somewhat singular $\dot{\Delta}(t)$ term in (9), the coefficient $g_{1}(t)$ in (6) is no longer of the order of the memory time $\tau$ but turns out to be of the same order as the zero order term $g_{0}$, and both terms in (4) are relevant in the $\tau \rightarrow 0$ limit. The forth line in (4) involving the function $g_{2}(t)$ vanishes for $L=L^{\dagger}$. Note that we have slightly changed our convention in order to be in line with standard notation: the correlation function (9) is here normalized such that the coefficient of the imaginary part, relevant for the damping, is unity.

Let us consider the high-temperature spin-boson model [12]. The system Hamiltonian is $H=-\frac{\omega}{2} \sigma_{x}+\frac{\Omega}{2} \sigma_{z}$, where $\omega$ is the tunneling matrix element and $\Omega$ depicts the bias of the system. The coupling operator is $L=\sqrt{\gamma} \sigma_{z}$. The 'post'-Markov master equation can be obtained directly from (价:

$$
\begin{aligned}
\dot{\rho}_{t}= & -i\left[H, \rho_{t}\right] \\
& +\gamma g_{0}(t) \sigma_{z} \rho_{t} \sigma_{z}-\gamma g_{0}(t) \rho_{t}+H . c . \\
& -i \omega \gamma g_{1}(t) \sigma_{x} \rho_{t}-\omega \gamma g_{1}(t) \sigma_{y} \rho_{t} \sigma_{z}+H . c .
\end{aligned}
$$

After an initial slip $(t \gg \tau)$, the $g_{i}(t)$ approach their asymptotic values and the density operator evolves according to

$$
\begin{aligned}
\dot{\rho}_{t}= & -i\left[H, \rho_{t}\right]+2 k T \gamma \sigma_{z} \rho_{t} \sigma_{z}-2 k T \gamma \rho_{t} \\
& +\omega \gamma\left\{\sigma_{x}, \rho_{t}\right\}-i \omega \gamma \sigma_{y} \rho_{t} \sigma_{z} \\
& +i \omega \gamma \sigma_{z} \rho_{t} \sigma_{y} .
\end{aligned}
$$

Obviously, both equation (10) and its asymptotic form (11) are not of Lindblad form (11) due to the presence of the last three terms. Therefore, as a Markov equation with constant coefficients, we cannot expect (11) to preserve the positivity of $\rho_{t}$ if applied to an arbitrary initial density operator. Our derivation shows that for the asymptotic equation (11) to be meaningful, one has to use an effective initial condition 咍 obtained by fully determining the initial slip arising from the time dependent coefficients $g_{i}(t)$ in the true master equation (10).

We show numerically that (10) preserves the positivity of the density matrix while the asymptotic equation (11) applied to the same initial condition fails to do so. The positivity of $\rho_{t}$ is equivalent to the condition $\|\langle\vec{\sigma}\rangle\| \leq 1$, where $\langle\vec{\sigma}\rangle=\operatorname{Tr}(\vec{\sigma} \rho)$ is the Bloch vector. In Fig. 2 we plot the norm of the Bloch vector using the time-dependent master equation (10) (solid curve) and using its asymptotic form (11) right from the start (dotted curve). Clearly, the latter loses positivity for some initial states on short time scales, whereas the full post-Markov master equation (10) preserves it for all times as numerically confirmed for a wide range of parameters in the region $k T \gg \Lambda=\tau^{-1}$, where $\Lambda$ is the cut-off frequency of the heat bath. Note however that if the correlation time is too large, then the post-Markov master equation can also produce non-positive results as should be expected from its derivation.

Note that this simple model is the 2-level analog of the quantum Brownian motion model where we have $H=$ $\frac{1}{2} p^{2}+V(q)$ and $L=\sqrt{\gamma} q$. The post-Markov master equation (4) for this case reads

$$
\begin{aligned}
\dot{\rho}_{t}= & -i\left[H, \rho_{t}\right]-\gamma g_{0 R}(t)\left[q,\left[q, \rho_{t}\right]\right]-i \gamma g_{0 I}(t)\left[q^{2}, \rho_{t}\right] \\
& +\gamma g_{1 R}(t)\left[q,\left[p, \rho_{t}\right]\right]+i \gamma g_{1 I}(t)\left[q,\left\{p, \rho_{t}\right\}\right]
\end{aligned}
$$


where the coefficients $g_{i R}(t), g_{i I}(t)$ are the real and imaginary parts of $g_{i}(t),(i=0,1)$, respectively. In the special case when $V(q)$ is a quadratic potential, it is reassuring that our master equation (12) coincides with the first order expansion of the exact Hu-Paz-Zhang master equation [5].

As in the previous example, for the high temperature Ohmic correlation function (9), the functions $g_{i}$ approach their asymptotic values after an initial slip on the time scale $\tau$ and (12) becomes the standard quantum Brownian motion (Caldeira-Leggett [13]) master equation

$$
\dot{\rho}_{t}=-i\left[H^{\prime}, \rho_{t}\right]-i \frac{\gamma}{2}\left[q,\left\{p, \rho_{t}\right\}\right]-\gamma k T\left[q,\left[q, \rho_{t}\right]\right]
$$

where $H^{\prime}$ is a cutoff-dependent renormalized Hamiltonian and where we dropped a term involving $\tau p$ with respect to a similar term proportional to $q$.

It is known that the non-Lindblad master equation (13) may violate the positivity of the density operator. Our full post-Markov master equation (12) is also a non-Lindblad equation but with time-dependent coefficients. As in the case of the spin-boson model, their time dependence can assure the preservation of the state's positivity for a wide range of parameters in the validity region of Eq. (10). In (12), the coefficient $g_{1 I}(t)$ of the dissipative term is zero at $t=0$ and its time derivative vanishes, too. The diffusion coefficient $g_{0 R}(t)$ also vanishes but its initial derivative is positive. Thus the initial phase of the evolution is dominated by diffusion. This mechanism, as is well known in the exact model of Ref. [月], may guarantee the positivity of the density matrix for short times as well as at later times when the dissipation enters. In contrast, the asymptotic $(t \gg \tau)$ Caldeira-Leggett master equation (13), if used right from the start $(t \geq 0)$, will immediately violate the positivity of a distinguished class of initial density matrices due to the constant dissipative term.

It is interesting to stress the close connection between the post-Markov master equation (何) and the QSD equation corresponding to (2) (see [10]). Indeed, the former was derived from the first order perturbation of the latter. Consequently, the post-Markov master equation (仿) can be simulated by using the powerfull quantum trajectory techniques provided by QSD [10].

To sum up, we have presented a post-Markov master equation for the evolution of open quantum systems. We have illustrated its great potential using several examples and we have further shown numerically that, for a wide range of parameters, the post-Markov master equation preserves the positivity of the density operator while its asymptotic version, disregarding the initial slip, fails to do so. This opens the road to applications of the post-Markov master equation (4) to phenomena, such as quantum decoherence and dissipation, photonic bandgaps, atom laser and more generally atom-field interactions in the weakly non-Markovian regimes.

We thank Ian C. Percival for useful suggestions and Bernard Gisin for help in numerical simulations. TY and NG would like to thank for support from the Swiss National Science Foundation and the European TMR Network "The physics of quantum information" (via the Swiss OFES). WTS thanks the Deutsche Forschungsgemeinschaft for support through the Sonderforschungsbereich 237 "Unordnung und große Fluktuationen".

[1] R. P. Feynman and F. L. Vernon, Ann. Phys. (N.Y.) 24, 118 (1963).

[2] R. Zwanzig, J. Chem. Phys. 33, 1338 (1960).

[3] S. Bay, P. Lambropoulos and K. Mølmer, Phys. Rev. Lett. 76, 161 (1996);J. J. Hope, Phys. Rev. A 55, R2531 (1997); S. John and T. Quang, Phy. Rev. Lett. 74, 3419(1994); N. Vats and S. John, Phys. Rev. A 58, 4168 (1998) and reference therein; G. M. Moy, J. J. Hope and C. M. Savage, Phys. Rev. A 59, 167 (1999).

[4] F. Haake and R. Reibold, Phys. Rev. A 32, 2462 (1985); S. Gnutzmann and F. Haake, Z. Phys. B 101, 263 (1996).

[5] B. L. Hu, J. P. Paz and Y. Zhang, Phys. Rev. D 45, 2843 (1992); Phys. Rev. D 47, 1576 (1993).

[6] J. J. Halliwell and T. Yu, Phys. Rev. D 53, 2012 (1996); L. Dávela Romero and J. P. Paz, Phys. Rev. A 55 , 4070 (1997).

[7] C. Anastopoulos and B. L. Hu, LANL e-print quant-ph/9901078 (unpublished).

[8] G. Lindblad, Comm. Math, Phys. 48, 119(1973).

[9] C. W. Gardiner, Quantum Noise (Springer, Berlin, 1991); H. J. Carmichael, Statistical Methods in Quantum Optics $I$ (Springer,1999).

[10] T. Yu, L. Diósi, N. Gisin and W. T. Strunz, LANL e-print quant-ph/9902043, Phys. Rev. A (in press).

[11] L. Diósi, N. Gisin, and W. T. Strunz, Phys. Rev. A 58, 1699 (1998); L. Diósi and W. T. Strunz, Phys. Lett. A 224, 25 (1996); W. T. Strunz, L. Diósi, and N. Gisin, Phys. Rev. Lett. 82, 1801 (1999).

[12] A. J. Leggett, S. Chakravarty, A. T. Dorsey, M. P. Fisher, A. Garg, and W. Zwerger, Rev.Mod. Phys. 59, 1(1987).

[13] A. O. Caldeira and A. J. Leggett, Physica A 121, 587 (1983). 


\section{CAPTIONS OF FIGURES}

FIG. 1. Illustration of the limit of the Markov approximation (dotted curve) for a damped two-level system. The dashed curve is our post-Markov result, the solid curve is the exact result. The initial state is $\left|\psi_{0}\right\rangle=|-\rangle$. The parameters are $\omega=\gamma, \gamma \tau=0.2$.

FIG. 2. Norm of the Bloch vector for the spin-boson model evolved with the post-Markov equation (10) (solid curve) and evolved with the asymptotic equation (11) (dotted curve). As the latter leads to a norm greater than one, it violates the positivity of $\rho_{t}$. The initial state is $\left|\psi_{0}\right\rangle=|+\rangle$ and the parameters are $\Omega=0, \omega \tau=0.01, k T \tau=20, \gamma=0.3 \omega$. 
Fig. 1

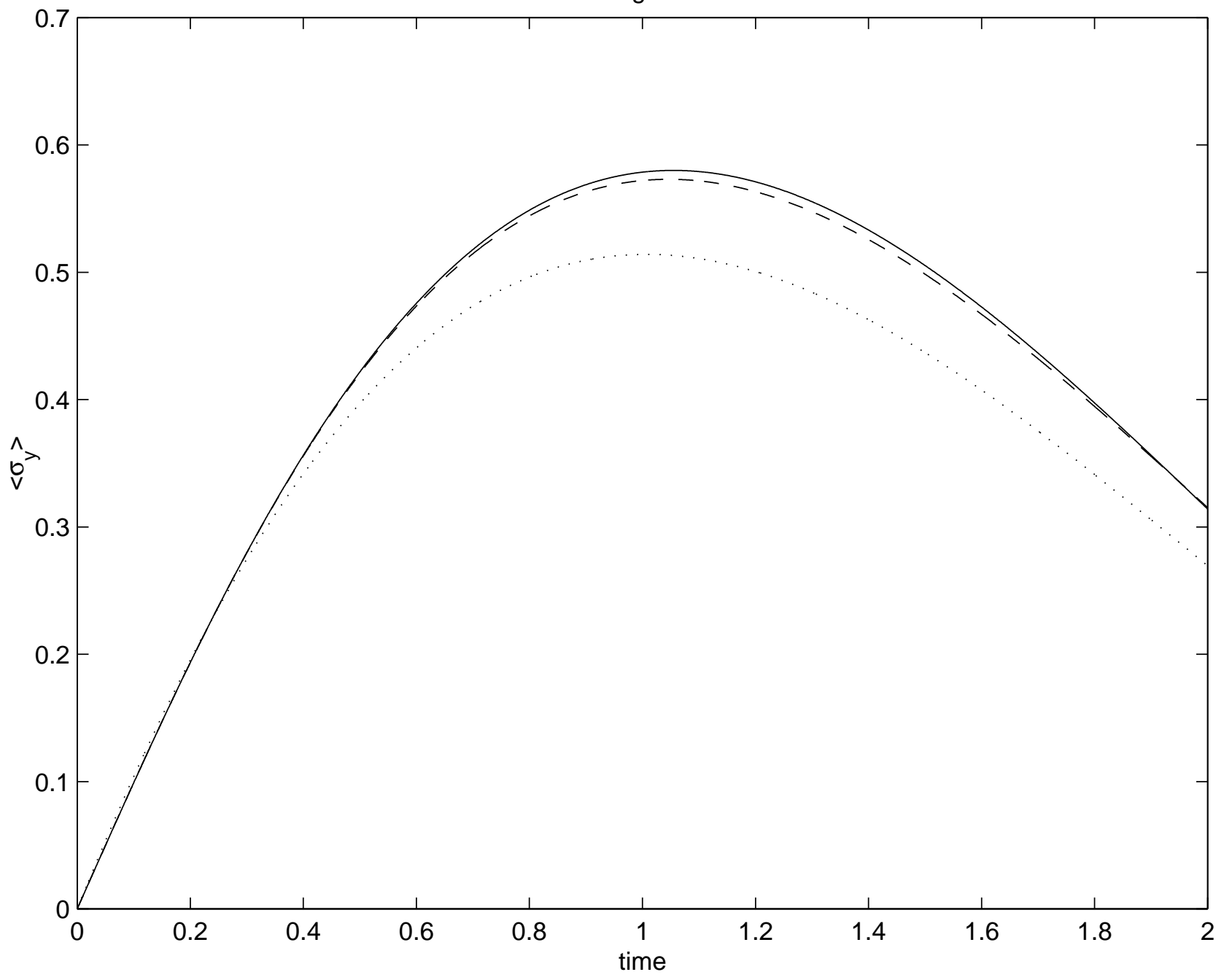




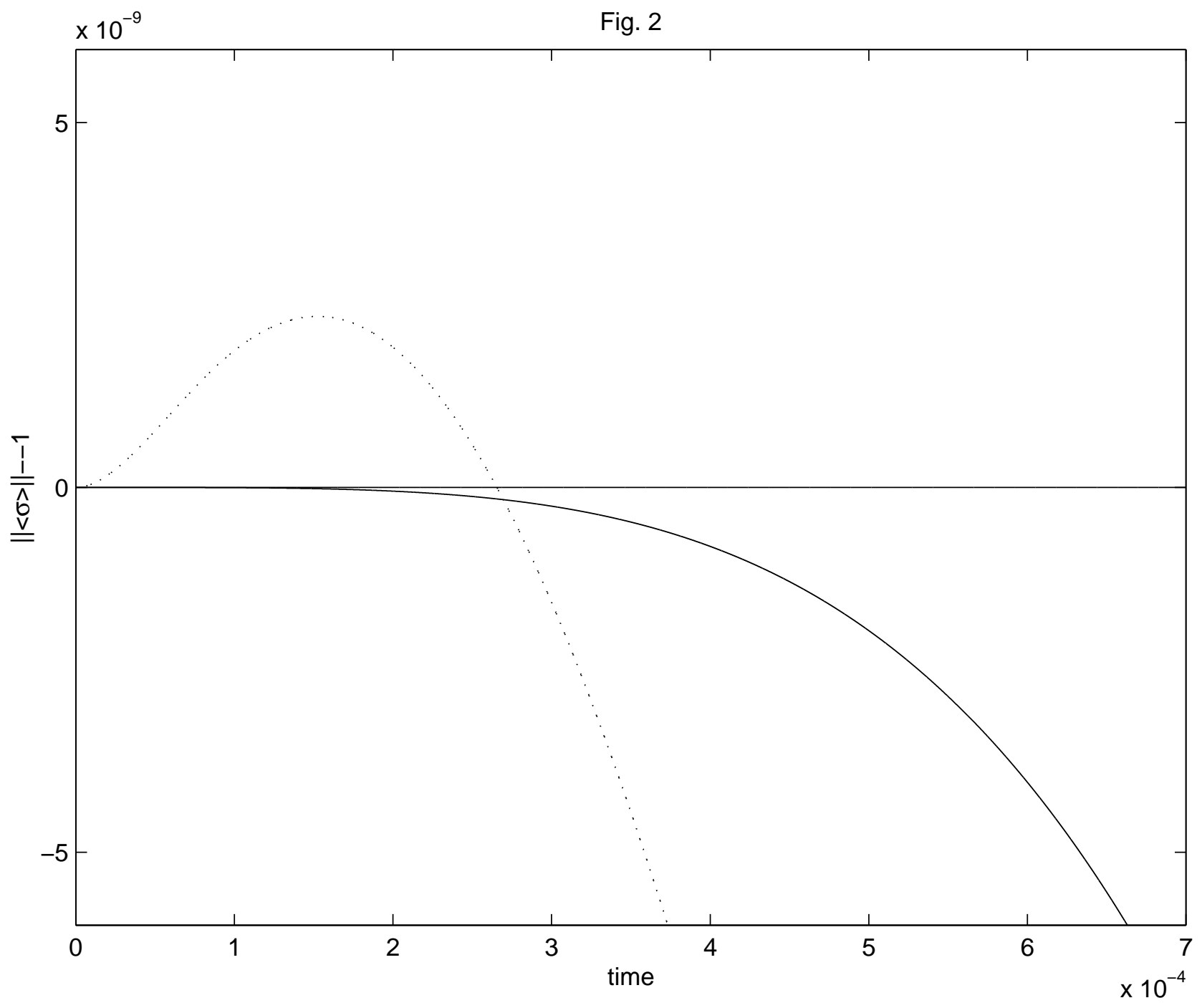

